\title{
Defect Depth Estimation Using Neuro-Fuzzy System in TNDE
}

\author{
by Akbar Darabi and Xavier Maldague
}

\begin{abstract}
Electrical Engineering Dept., Université Laval, Quebec City (Quebec) Canada G1K 7P4, E-mail:
\end{abstract} darab@gel.ulaval.ca and maldagx@gel.ulaval.ca

\section{Abstract:}

Recently, supervised artificial neural networks have obtained success to reveal and provide quantitative information concerning defects in TNDE (Thermographic NonDestructive Evaluation). Supervised neural networks may converge to local minimum and their training procedure are usually long. In this study, a neuro-fuzzy approach is applied to characterize subsurface defects in TNDE. Similar to neural networks, fuzzy systems are model-free estimator systems which can learn from experience with numerical or linguistic data. In this paper, the concept of a fuzzy set and fuzzy reasoning mechanisms are first discussed. Then, a neuro-fuzzy defect depth estimator based on the Takagi-Sugeno-Kang (TSK) system modeling method is proposed. Finally, the neuro-fuzzy depth estimator is tested with both simulated and experimental TNDE data.

\section{Introduction}

Similar to neural networks, fuzzy systems estimate a function without a mathematical model of how outputs depend on input data. This property gives opportunity to these systems to learn from experience with numerical or linguistic data. This classifies them as model-free estimator systems. Fuzzy systems are based on fuzzy sets which were first proposed in 1965 by Lotfi A. Zadeh [1]. Fuzzy sets are extended forms of conventional "Boolean" sets that can handle the concept of partially true values between "completely true" and "completely false", to deal with vagueness and uncertainty related to human linguistic and thinking principles of every day life [1], [2], [3], [4].

Neuro-Fuzzy computing approaches to system modeling have also attracted the attention of many researchers in the past several years [5]. This attraction is based on two key advantages of neuro-fuzzy systems. First, comparing to traditional quantitative modeling techniques, these systems are model-free and they do not require a mathematical description of the system. On the other hand, neuro-fuzzy methods posses both the low-level learning and computational power of neural networks and advantages of high-level humanlike thinking of fuzzy systems [6], [7].

To build-up a fuzzy system, first one should provide linguistic variables in place of or in addition to numerical variables. Then, the system requires some IF-THEN fuzzy rules to characterize simple relations between fuzzy variables. Finally, complex relationships should be established by fuzzy reasoning algorithms for the proposed fuzzy system. In general, fuzzy system modeling can be classified in two basic types. The first type was pioneered by Mamdani and Asilian in 1975. This system was applied to the regulation of a steam engine. The antecedent and consequent variables of IF_THEN rules are fuzzy in this system. This kind of model is also considered as a qualitative system modeling which uses human-like language expression [8]. The second type of fuzzy system modeling method, uses fuzzy inputs and rules but its outputs are non-fuzzy sets. This type of fuzzy model was initially proposed by Takagi and Sugeno [9]. It provides a powerful tool for the modeling of complex nonlinear problems.

\section{Fuzzy Logic}

Fuzzy set contains objects that satisfy imprecise properties of their membership functions. Sets such as children, young, or old people are examples of a fuzzy sets. In these examples the boundary of each set is not clearly defined. Lets, consider the young people set. It is difficult to classify people into "young" or not "young" people class. To being young is matter of degree that can vary from teenagers to old people. To express the degree of being a child, young or old, we use a membership function. The membership function for any fuzzy set maps sets objects into unit interval $[0,1]$. Value "0" significates the object does not 
belong to the set and value " 1 " corresponds to the object completely matching the set. There is no unique membership function for any fuzzy set since it depends on applications and properties which are desired for that set. Suppose $A$ is a fuzzy set in universe $X$ with membership function $m_{A}$. For each object, $x$ in $A$, the value of $m_{A}(x)$ represents the grade of membership of $x$ in $A$. If $A$ and $B$ are two fuzzy sets, the fuzzy set operations can be defined as follows:

- Union:

$$
\forall x \in X
$$

- Intersection: $\quad A \cap B \Leftrightarrow m_{A \cap B}(x)=\min \left(m_{A}(x), m_{B}(x)\right)$

- Complement:

$$
m_{\bar{A}}(x)=1-m_{A}(x)
$$

- Equality:

$$
A=B \Leftrightarrow m_{A}(x)=m_{B}(x)
$$

- and Inclusion:

$$
A \subset B \Leftrightarrow m_{A}(x) \leq m_{B}(x)
$$

\section{Fuzzy Reasoning Mechanism}

In fuzzy modeling, after the input variables and their membership functions defined, some inference rules (if-then rules) to perform fuzzy reasoning are needed. In general, two basic approaches are used to infer if-then rule in most applications of the fuzzy modeling systems. The fuzzy reasoning methods can be classified as the "traditional-fuzzy" modeling method, in which an a priori knowledge about the system is available, and the "classical-fuzzy" method which is based on the use of an input-output data relationship. The most popular of the traditional fuzzy reasoning method was first proposed by Mamdani and Asilian [8]. Additional to the input variables which are fuzzy sets for both reasoning methods, this inference method expects the output membership functions to be also fuzzy sets. The relations between fuzzy variables are given by composed conditional statements (if-then rules) which use the union or intersection operators that are called min-max operators. The general form of the Mamdani fuzzy system rule using "and" operator is expressed by:

$$
\boldsymbol{R}^{i} \text { : If } x_{1} \text { is } X_{1}^{i} \text { and } x_{2} \text { is } X_{2}^{i}, \ldots \text {, and } x_{n} \text { is } X_{n}^{i} \text { then } y \text { is } Y_{i} \text {. }
$$

Where $\mathbf{R}^{i}$ is $i^{\text {th }}$ rule of the system, $X_{1}, X_{2}, \ldots$, and $x_{n}$ are input fuzzy variables, $X_{1}^{i}, X_{2}^{i}, \ldots, X_{n}^{i}$ are input fuzzy sets, $y$ is the output variable and $Y_{j}$ is the $i^{\text {th }}$ rule corresponding to the output fuzzy set. The true value of the antecedent part of $\mathrm{i}^{\text {th }}$ rule is derived from Eq. 6 using Eq. 2 as:

$$
w_{i}=\min \left(m_{X_{1}^{i}}\left(x_{1}\right), m_{X_{2}^{i}}\left(x_{2}\right), \ldots, m_{X_{n}^{i}}\left(x_{n}\right)\right)
$$

where $w_{i}$ is the single value result of antecedent part that should be applied to the consequent part to get the $i^{\text {th }}$ rule output value. Eq. 7 measures the adaptability of $i^{\text {th }}$ rule to the input $\left(x_{1}, x_{2}, \ldots, x_{n}\right)$ and applies that result to the consequent part. This is know as an implication. The implication process in fuzzy logics theory is similar to the binary logic. In binary logic the antecedent and consequent of an implication are either true or false but fuzzy theory permits the partial truth in antecedent and consequent part of fuzzy implication such as:

$$
0.5 p \rightarrow 0.5 q
$$

where $p$ and $q$ are the antecedent and consequent part variables of the implication. Therefore to obtain the output of each rule we should project the adaptability result to the consequent part so that a fuzzy set is assigned to the output. The conclusion of the $i^{\text {th }}$ rule is then given as:

$$
m_{Y_{i}^{\prime}}(y)=\min \left(w_{i}, m_{Y_{i}}(y)\right) \quad \forall y \in Y
$$

where $Y_{i}^{\prime}$ is the conclusion fuzzy set of $i^{\text {th }}$ rule and $Y$ is the output universe. From aggregation of each rule conclusion fuzzy set, we can then obtain the final conclusion fuzzy set as:

$$
m_{Y^{\prime}}(y)=\max \left(m_{Y_{1}^{\prime}}(y), m_{Y_{2}^{\prime}}(y), \ldots, m_{Y_{n}^{\prime}}(y)\right) \quad \forall y \in Y .
$$


In Eq. $9 Y^{\prime}$ is called the aggregated output of the fuzzy system for a given input. Here, the "max" operand is chosen as aggregation process, but one can use any other functions depending on the application. To obtain a single output value, we need to defuzzify the aggregated fuzzy set. There are different methods of defuzzification such as min, max, middle and centroid calculation methods. The most popular defuzzification method is the centroid calculation which is given by [4]:

$$
y_{0}=\int y m_{Y^{\prime}}(y) d y / \int m_{Y^{\prime}}(y) d y
$$

where $y_{0}$ is the single value output of the system.

\section{Neuro-Fuzzy Defect Depth Estimation}

The conventional fuzzy system modeling uses a single model to describe the global behavior of a system. This kind of fuzzy system modeling needs a large number of rules to represent the behavior of a sophisticated system. The Takagi-Sugeno-Kang (TSK) system modeling method divides the input space into subspaces and gives a simple model for each input subspace which are called submodels of the system [9], [10]. The combination of these submodels constitutes the global behavior of the system. On the other hand, since the consequent part of implications are explicitly expressed by the input variables, some learning algorithms can be employed to identify system parameters [11]. Several approaches have been proposed to generate fuzzy if-then rules from training data based on TSK fuzzy model. One such an approach is called the Adaptive-Network-Based Fuzzy Inference System (ANFIS) [12]. ANFIS is a class of adaptive multi-layer feed-forward networks that is functionally equivalent to a fuzzy inference system. Each neuron in ANFIS applies a particular function on incoming signals as well as a set of relating parameters to each neuron. To identify the adaptive network parameters, this fuzzy inference method employes a hybrid learning algorithm which combines the gradient method and the least squares estimate (LSE). Not only can this hybrid learning algorithm guarantees to find global minima but it also cut down the convergence time of the network due to decreasing dimensions of the research space in the gradient method [12].

ANFIS can be applied to complex nonlinear problems such as defect depth estimation in TNDE. This section is dedicated to defect depth estimator using ANFIS. As discussed earlier the number of rules augments exponentially while increasing the number of input variables to the system. For example, if we consider thermal contrast vectors with 16 sampled points for each vector as inputs to the system and suppose two labels for each sampled point, then the ANFIS requires $2^{16}$ initial rules! This complicates the reasoning process and is beyond fuzzy system modeling goals. Therefore, the system should be provided with less characteristic data points. It is reported that the defect depth can mathematically be expressed as a function of the maximum contrast and its occurrence time. Such a relation for graphite epoxy specimens is given by [13]:

$$
Z_{\text {def }}=0.6722 \sqrt{t_{C \max }}\left(C_{\max }\right)^{-0.258}
$$

Where $Z_{\text {def }}$ is defect depth, $t_{C \max }$ is time of the maximum contrast and $C_{\max }$ is maximum contrast on the specimen surface.

In our case, we will provide four additional informative parameters such as time of halfrise contrast $t_{1 / 2 C \max }$, time of half- decay $t^{+}{ }_{1 / 2 C \max }$ and their related contrast to the ANFIS depth estimator. These parameters are shown in Figure 1 for a simulated Teflon ${ }^{\mathrm{TM}}$ defect at depth $0.7 \mathrm{~mm}$ under the surface. The difference between half-rise and half-decay contrasts is due to the sampling time. Next we propose two ANFIS depth estimators, one for the CFRP samples containing Teflon ${ }^{\mathrm{TM}}$ defects and the other for samples with air defects. First, the ANFIS depth estimators are provided with training vectors as shown in Figure 2. Each

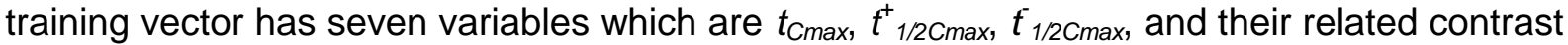
as inputs. and depth corresponding to the chosen pixel as the output of the estimator.

If we assign two membership functions to each input variables, the ANFIS systems have 
then 64 rules. The initial membership function for each input variable is two equally spaced bell-shaped functions with enough overlap. The training process takes about 2 minutes on a Sun workstation (ULTRA 30). The shape of the membership functions changes during the training process to minimize the output error of the estimator.

\section{RESUTS}

To examine the air defect, with the ANFIS depth estimator, we take the input data extracted from a simulated CFRP sample containing a T-shape air defect. The estimated depth profiles without any post processing are shown in Figure 3 (b) and Figure 5 . These figures show that the ANFIS depth estimator is able to estimate simulated defect depths as the neural network depth estimator proposed in [14]. If the detected error pixels are removed, the estimated depth for defect pixels becomes as illustrated in Figure 3 (c) and Figure 4. Comparing this figure with original depth profiles shown in Figure 3 (a) confirms precision and reliability of the ANFIS depth estimators. The estimated depth profile error is depicted in Figure 3 (d) and the estimated depth profile error in which the error pixels have been removed is shown in Figure 3(e). The average relative error is $8.66 \%$.

The available simulated data for the CFRP samples containing Teflon ${ }^{\mathrm{TM}}$ defects are only valid for defects at depth greater than $1 \mathrm{~mm}$ depth due to Maspar computer which is now out of operation in our laboratory, so that we could not modify the set-up parameters to have new reliable simulated data for the training process [14]. For the experimental part of this project, We thus have access to limited data to work with CFRP samples containing Teflon ${ }^{\mathrm{TM}}$ defects. Nevertheless we used the available data to train the Teflon ${ }^{\mathrm{TM}}$ defect ANFIS depth estimator.

After training the Teflon ${ }^{\mathrm{TM}}$ defect ANFIS depth estimator, we provided as inputs, data extracted from real IR images in the case of a CFRP sample with two rectangular Teflon ${ }^{T M}$ defects. The ANFIS depth estimator output is shown in Figure 6. Although, the ANFIS depth estimator reveals both defects and estimated their depth, there is still a lot of noise that limits visualization of the estimated depth profiles over defects. Therefore, the output is filtered using a median mask and depth profiles are represented in Figure 7. This figure shows that the estimator classify some other than defect region pixels as defect. This is due to both non-uniform heating of the sample surface and non-homogeneity of thermal properties in this CFRP sample. Nevertheless, the estimated depth over defect region somehow agrees with the result of estimated depth by the neural network depth estimator. Moreover, it seems that the defects are revealed more clearly by the ANFIS depth estimator as compared with the neural network depth estimator results in [14].

\section{Conclusion}

We discussed the concept of fuzzy sets and demonstrated their advantages for data interpretation and classification as compared with crisp set theory. Two fuzzy system modeling approaches, Mamdani and TSK fuzzy models, were reviewed. TSK model permits to divide complex problems to simple submodels where in each submodel a linear relation can simply be established between its premis and consequence part. We also described how an adaptive-network based fuzzy inference system (ANFIS) can employ an hybrid learning algorithm to define if-then rules for a complex system expressed with a training set. Finally we proposed a neuro-fuzzy depth estimator based on ANFIS system modeling. The proposed defect depth estimator was tested on simulated and real experimental data. The results showed this new approach brings both accuracy and short training time for quantitative thermal testing method.

\section{Acknowlegements}

The Ministry of Science, Research and Technology of the Islamic Republic of Iran and NSERC (Canada) are thanked for the support to this work. CNR (Italy) is thanked for providing the CFRP specimen. 


\section{References}

[1] L. A. Zadeh, "Fuzzy sets". Inform. Control, Vol. 8, pp 338-353, 1965

[2] L. A. Zadeh, "Outline of a New Approach to the Analysis of Complex System and Decision Processes", IEEE Trans. Syst. Man, Cybern., vol. 3, pp. 28-44, 1973.

[3] B. Kosko, "Neural Networks and Fuzzy Systems", Englewood Cliffs, NJ: PrenticeHall, 1992.

[4] K. Tanaka, "An Introduction to Fuzzy Logic for Practical Applications", SpringeVerlag, 1997

[5] M. R. Emami, I. B. Türksen, and A. A. Goldenberg, "Development of A Systematic Methodology of Fuzzy Logic Modeling", IEEE Trans. Fuzzy Syst. vol. 6, no. 3, pp. 346-361 Agu. 1998.

[6] M. Sugeno and T. Yasukawa, "A Fuzzy-Logic-Based Approach to Qualitative Modeling", IEEE Trans. Fuzzy Syst. vol. 1, no. 1, pp. 7-31 Feb. 1993.

[7] C. F. Juang and C. T. Lin, "an On-Line Self-Construction Neural Fuzzy Inference Network and Its Applications", IEEE Trans. Fuzzy Syst. vol. 6, no. 1, pp. 12-32 Feb. 1998.

[8] E. Mamdani and S. Asilian, "An Experiment in Linguistic Synthesis with a Fuzzy logic Controller", International Journal of Man Machine Studies, vol. 7, pp 1-13, 1975.

[9] T. Takagi and M. Sugeno, "Fuzzy identification of systems and its application to modeling and control", IEEE Trans. Syst. Man, Cybern., vol. SMC-15, pp. 116-132, Jan. 1985.

[10] M. Sugeno and G. T. Kang, "Structure identification of fuzzy model", Fuzzy Sets Syst., vol. 28, pp. 15-33 1988.

[11] J. Yen, L. Wang and C. W. Gillespie, "Improving the interpretability of TSK fuzzy models by combining global learning and local learning", IEEE Trans. Fuzzy Syst. vol. 6, no. 4, pp. 530-537 Nov. 1998.

[12] J. S. R.r Jang, "ANFIS: Adaptive-Network-Based Fuzzy Inference System", IEEE Trans. Syst. Man, Cybern., vol. 23, No. 3, pp. 665-685, May/June. 1993.

[13] X. Maldague, "Theory and Practice of Infrared Technology for Non Destructive Testing," Wiley-Interscience, New York, 2001 [in press].

[14] A. Darabi and X. Maldague, "Neural Network Based Defect Detection and Depth Estimation in TNDE," Submitted to Canadian Society of NonDestructive Testing Journal, July 2000.

Figure1: The normalized contrast curve on the surface of a CFRP sample over a Teflon $^{\mathrm{TM}}$ subsurface defect at $0.7 \mathrm{~mm}$ depth.

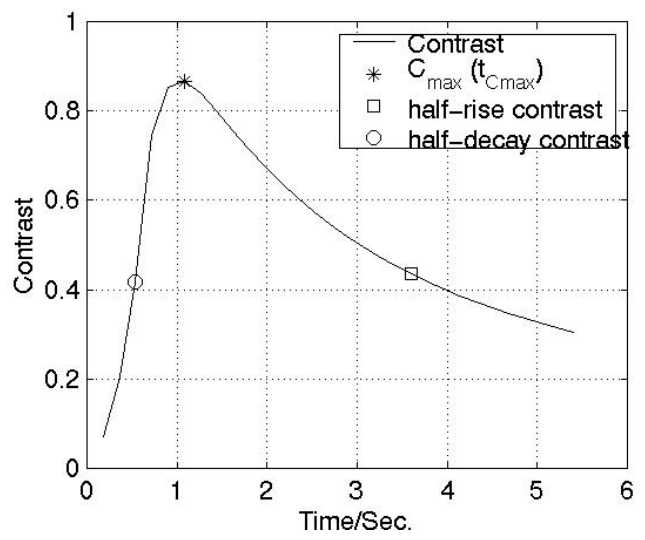




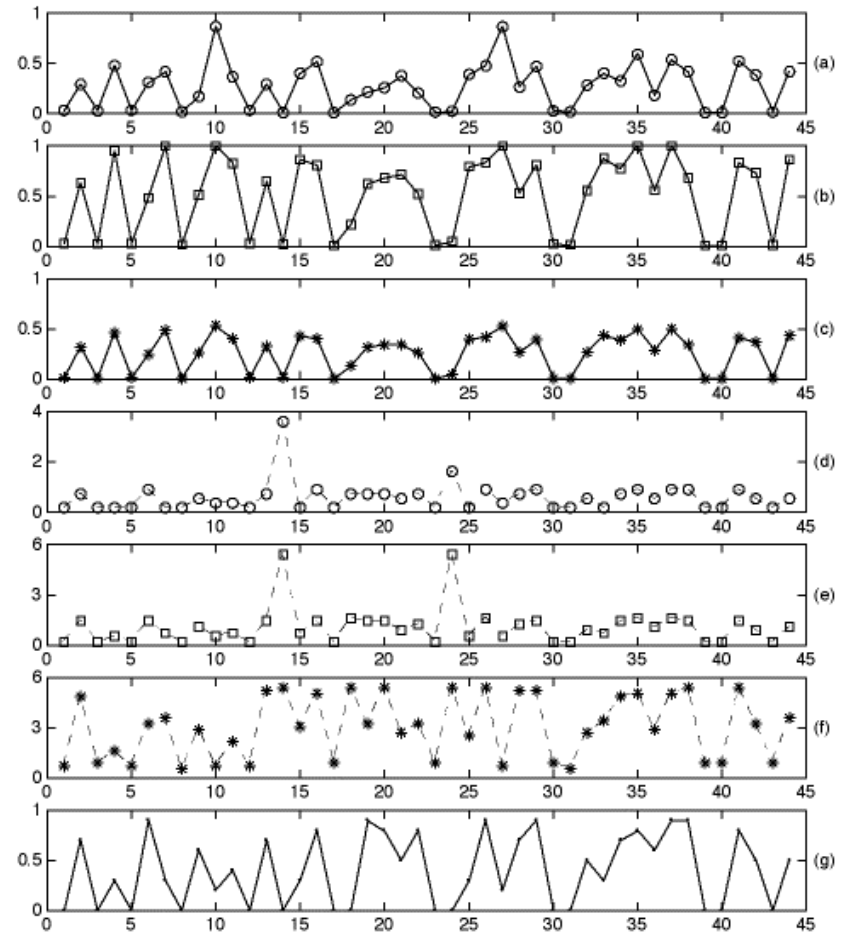

Figure 2: Training set for ANFIS depth estimator. The vectors extracted from simulated data of CFRP samples containing Teflon ${ }^{\mathrm{TM}}$ defects at various depths. (a) halfrise contrast (b) maximum contrast (c) half-decay contrast (d) half-rise contrast time (e) maximum contrast time (f) half-decay contrast time (g) depth.
Depth/mm

(a)

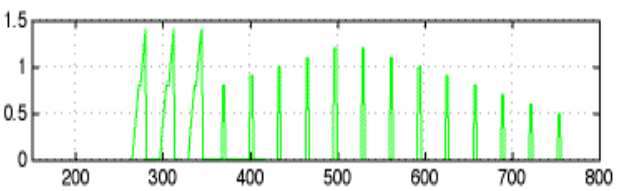

(b)

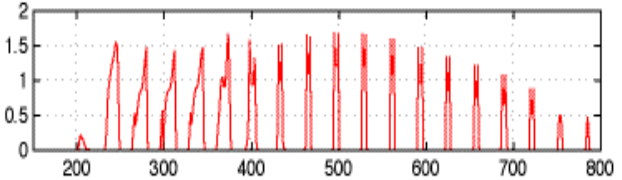

( c )
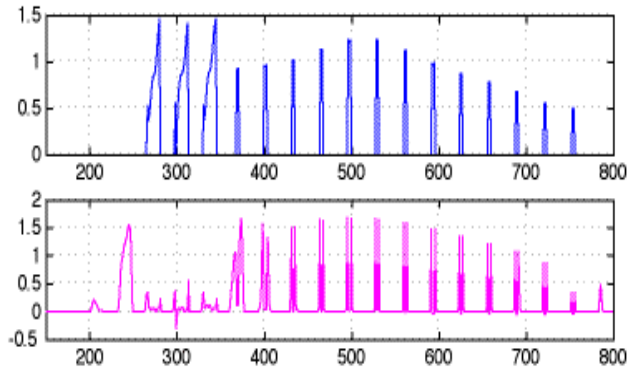

(e)

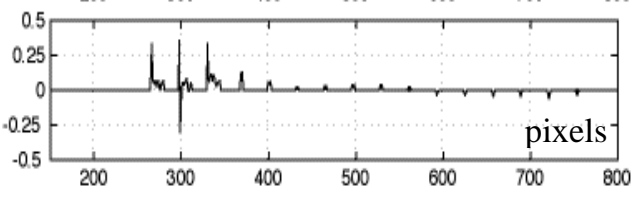

Figure 3: Depth profile for T-shape air defect buried in CFRP sample. (a) Original depth (b) ANFIS estimated depth (c) the ANFIS estimated depth in which error defect pixels have been removed (d) depth error (e) the depth error in which error defect pixels have been removed.

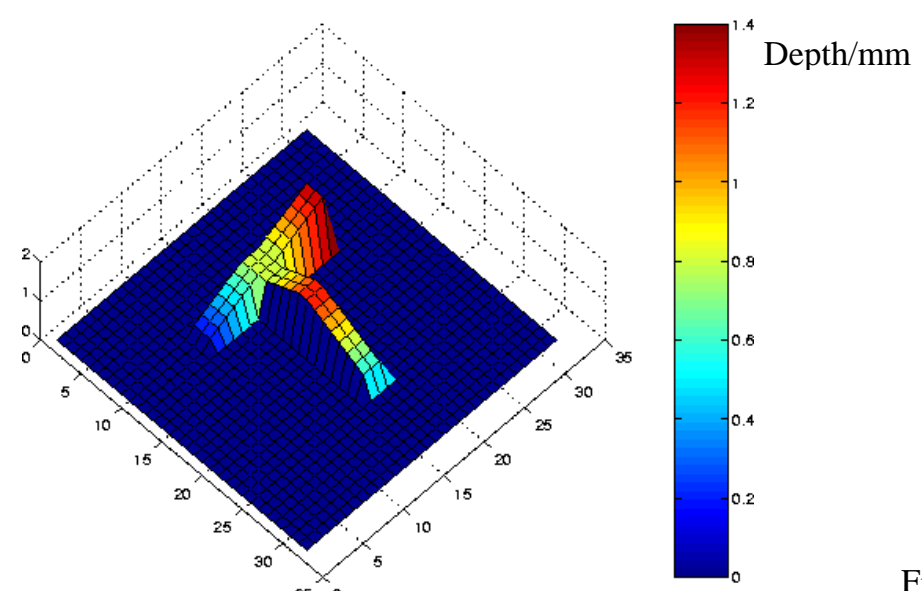

Figure 4: 3-D depth profile of a T-shaped air defect buried in CFRP sample.

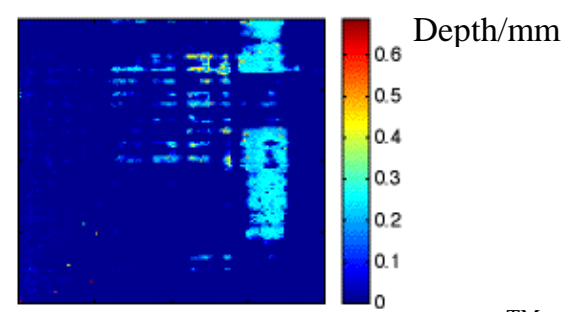

Figure 6: Estimated depth image of Teflon ${ }^{\mathrm{TM}}$ defect by ANFIS depth estimator for an CFRP sample.

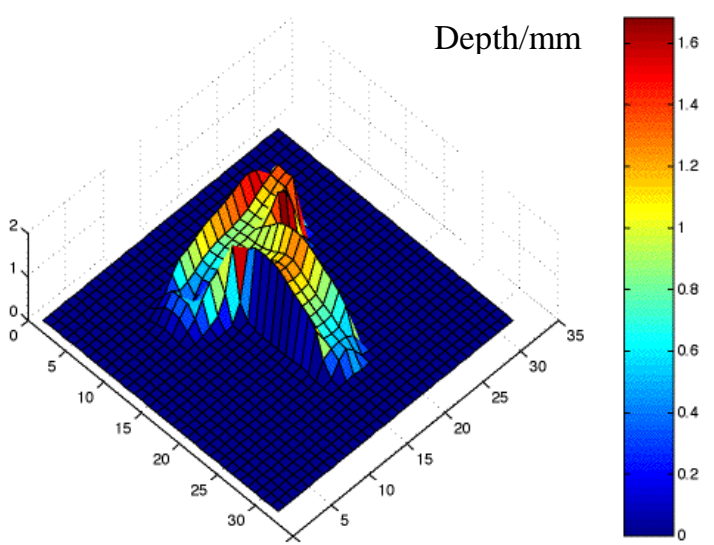

Figure 5: 3-D estimated depth profile of the subsurface air defect ANFIS depth estimator for Tshaped air defect buried in CFRP sample.

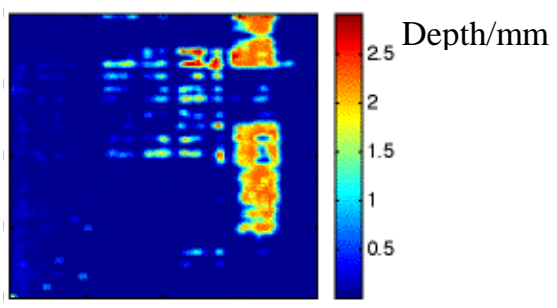

Figure 7: The filtered depth estimation image of Teflon $^{\mathrm{TM}}$ defect by ANFIS depth estimator for an CFRP sample . 
http://dx.doi.org/10.21611/qirt.2000.032 